\title{
Effects of Axion-Photon Mixing on Gamma-Ray Spectra from Magnetized Astrophysical Sources
}

\author{
Kathrin A. Hochmuth ${ }^{1}$ and Günter Sigl ${ }^{2}$ \\ ${ }^{1}$ Max-Planck-Institut für Physik (Werner-Heisenberg-Institut), Föhringer Ring 6, 80805 München, Germany \\ ${ }^{2} A P C^{*}$ (AstroParticules et Cosmologie), 10, rue Alice Domon et Léonie Duquet, 75205 Paris Cedex 13, France \\ and Institut d'Astrophysique de Paris, UMR 7095 CNRS - Universite \\ Pierre \& Marie Curie, 98 bis boulevard Arago, F-75014 Paris, France
}

\begin{abstract}
Astrophysical $\gamma$-ray sources come in a variety of sizes and magnetizations. We deduce general conditions under which $\gamma$-ray spectra from such sources would be significantly affected by axionphoton mixing. We show that, depending on strength and coherence of the magnetic field, axion couplings down to $\sim\left(10^{13} \mathrm{GeV}\right)^{-1}$ can give rise to significant axion-photon conversions in the environment of accreting massive black holes. Resonances can occur between the axion mass term and the plasma frequency term as well as between the plasma frequency term and the vacuum CottonMouton shift. Both resonances and non-resonant transitions could induce detectable features or even strong suppressions in finite energy intervals of $\gamma$-ray spectra from active galactic nuclei. Such effects can occur at $\mathrm{keV}$ to $\mathrm{TeV}$ energies for couplings that are currently allowed by all experimental constraints.
\end{abstract}

PACS numbers: 98.70.Rz, 14.80.Mz, 98.54.Cm, 98.58.Fd

\section{INTRODUCTION}

Axions and axion-like particles couple to two photons and can thus convert into on-shell real photons and viceversa in the presence of magnetic fields. Astrophysical sources produce high energy $\gamma$-rays as secondary particles of primary charged cosmic rays. Since these primary cosmic rays have to be accelerated in electromagnetic fields, the spectrum of any astrophysical $\gamma$-ray source can potentially be modified by axion-photon mixing. In the present paper we discuss in detail in which source environments and for which axion masses and coupling constants one can expect significant modifications of the observable $\gamma$-ray spectrum. As specific examples we consider the jets and central engines of active galactic nuclei (AGN) both of which are driven by magnetized accretion disks.

We consider the Lagrangian for the coupling between a pseudo-scalar field $a$, called axion in the following, and the electromagnetic field strength $F_{\mu \nu}$

$$
\mathcal{L}_{\gamma a}=-\frac{1}{4} g_{\gamma a} F_{\mu \nu} \tilde{F}^{\mu \nu} a=g_{\gamma a} \mathbf{E} \cdot \mathbf{B} a,
$$

where $\tilde{F}_{\mu \nu} \equiv \frac{1}{2} \varepsilon_{\mu \nu \rho \sigma} F^{\rho \sigma}$ is the electromagnetic dual, $\mathbf{E}$ and $\mathbf{B}$ are the electric and magnetic field strengths, respectively, and $g_{\gamma a}$ is the photon-axion coupling. Eq. (1) implies that a photon can convert into an axion in a magnetic field $\mathbf{B}$. In the present work we will consider the effect of such conversions on $\gamma$-ray fluxes emitted from magnetized sources for general pseudo-scalar masses $m_{a}$ and photon-axion couplings $g_{\gamma a}$.

*UMR 7164 (CNRS, Université Paris 7, CEA, Observatoire de Paris)
Axions with couplings $g_{\gamma a} \lesssim 10^{-10} \mathrm{GeV}^{-1}$ are consistent with all existing constraints for almost all axion masses [1] and we will use $g_{\gamma a}=10^{-11} \mathrm{GeV}^{-1}$ as our benchmark value. As possible $\gamma$-ray sources we consider already detected sources, like Makarian 501 and Makarian 421. Sources of this type can have a model-dependent magnetic field strength between $0.01 \mathrm{G}$ and several $\mathrm{G}$. Adopting these values we find that a significant amount of $\gamma$-rays can be converted into axions, thereby leaving characteristic signatures like steps or gaps in the spectrum. This conversion can take place due to normal oscillations and due to resonance effects. We find that for typical parameters the effects can be sizable and are well within the detection region of the next generation of experiments.

Our paper is organized as follows. In Sec. II we discuss in general how the $\gamma$-ray spectra can be modifed due to resonant and non-resonant oscillation effects. We discuss the prospects of detecting these effects in Sec. III] where we discuss specifically the case of central engines and jets of AGN. In Sec. [IV we present our conclusions. We use natural units, $\hbar=c=k=1$, throughout the paper.

\section{CONDITIONS FOR MODIFICATION OF GAMMA-RAY SPECTRA}

The axion-photon conversion probability in a transverse magnetic field $B_{\mathrm{t}}$ can be derived by using the linearized equation of motion following from Eq. (1) for relativistic axions. This equation can be written as [2]

$$
\left(E-\mathrm{i} \partial_{z}-\mathcal{M}\right)\left(\begin{array}{c}
A_{\perp} \\
A_{\|} \\
a
\end{array}\right)=0,
$$

where $z$ is the direction of propagation, $E$ is the photon energy and $a$ the axion field. $A_{\perp}$ and $A_{\|}$are orthogonal 
components of the photon field, where $i=\perp$ or $\|$ refer to the $B_{\mathrm{t}}$ direction. The mixing matrix $\mathcal{M}$ is

$$
\mathcal{M} \equiv\left(\begin{array}{ccc}
\Delta_{\perp} & \Delta_{\mathrm{R}} & 0 \\
\Delta_{R} & \Delta_{\|} & \Delta_{B} \\
0 & \Delta_{B} & \Delta_{a}
\end{array}\right),
$$

where

$$
\begin{aligned}
\Delta_{\perp}=\Delta_{\mathrm{pl}}+\Delta_{\mathrm{CM}}^{\perp}, & \Delta_{B}=\frac{1}{2} g_{a \gamma} B_{\mathrm{t}} \\
\Delta_{\|}=\Delta_{\mathrm{pl}}+\Delta_{\mathrm{CM}}^{\|}, & \Delta_{\mathrm{pl}}=\omega_{\mathrm{pl}}^{2} /(2 E) .
\end{aligned}
$$

Here we have defined $\omega_{\mathrm{pl}}^{2}=4 \pi \alpha n_{e} / m_{e}$ as the plasma frequency for an electron density $n_{e}$, where $m_{e}$ is the electron mass and $\alpha \equiv e^{2} /(4 \pi)$ the fine-structure constant with $e$ the electron charge. $\Delta_{\mathrm{R}}$ is the Faraday rotation term, which is dependent on the energy of the longitudinal component of the magnetic field and couples to the modes $A_{\|}$and $A_{\perp}$. However, we want to consider only non-polarized sources, which renders this term negligible. The vacuum Cotton-Mouton effect is represented by the $\Delta_{\mathrm{CM}}$ terms, which describe the birefringence of fluids in presence of a longitudinal magnetic field, with $\left|\Delta_{\mathrm{CM}}^{\|}-\Delta_{\mathrm{CM}}^{\perp}\right| \propto B_{\mathrm{t}}^{2}$. Note that the photon dispersion relation for polarization $i$ is $E_{i}(k)=k+\Delta_{i}$, corresponding to the refractive indices $n_{i}-1 \simeq \Delta_{i} / E$. For axions one has, of course, $E(k) \simeq k+m_{a}^{2} /(2 k)$ and thus $\Delta_{a} \simeq m_{a}^{2} /(2 E)$.

Neglecting different photon polarization states and denoting the resulting photon state by $A$, we are left with a simple two-component mixing problem,

$$
\left[E-\mathrm{i} \partial_{z}-\left(\begin{array}{cc}
\Delta_{\mathrm{pl}}(z)+\Delta_{\mathrm{CM}}(z) & \Delta_{B}(z) \\
\Delta_{B}(z) & \Delta_{a}
\end{array}\right)\right]\left(\begin{array}{c}
A \\
a
\end{array}\right)=0,
$$

where we have indicated the terms that are location dependent. After diagonalization of the mixing matrix we obtain the solution

$$
\theta(z)=\frac{1}{2} \arctan \left(\frac{2 \Delta_{B}(z)}{\Delta_{\mathrm{pl}}(z)+\Delta_{\mathrm{CM}}(z)-\Delta_{a}}\right) .
$$

Within a domain of linear size $s$ and roughly constant plasma density and magnetic field, the probability of a transition from a photon to an axion is [2, 3, 4]

$$
P_{\gamma \rightarrow a} \simeq\left(\Delta_{B} s\right)^{2} \frac{\sin ^{2} \Delta_{\mathrm{osc}} s / 2}{\left(\Delta_{\mathrm{osc}} s / 2\right)^{2}} \equiv P_{0},
$$

where the oscillation wave number is

$$
\Delta_{\mathrm{osc}}^{2} \simeq\left(\Delta_{\mathrm{CM}}+\Delta_{\mathrm{pl}}-\Delta_{a}\right)^{2}+4 \Delta_{B}^{2} .
$$

In Eq. (8), the plasma contribution $\Delta_{\mathrm{pl}}$, the vacuum Cotton-Mouton term $\Delta_{\mathrm{CM}}$, the axion mass term $\Delta_{a}$, and the off-diagonal mixing term $\Delta_{B}$ are given by

$$
\Delta_{a}=\frac{m_{a}^{2}}{2 E} \simeq 2.5 \times 10^{-20} m_{\mu \mathrm{eV}}^{2}\left(\frac{\mathrm{TeV}}{E}\right) \mathrm{cm}^{-1},
$$

$$
\begin{aligned}
\Delta_{\mathrm{pl}} & =\frac{\omega_{\mathrm{pl}}^{2}}{2 E} \simeq 3.5 \times 10^{-26}\left(\frac{n_{e}}{10^{3} \mathrm{~cm}^{-3}}\right)\left(\frac{\mathrm{TeV}}{E}\right) \mathrm{cm}^{-1}, \\
\Delta_{\mathrm{CM}} & \simeq-\frac{\alpha}{45 \pi}\left(\frac{B_{\mathrm{t}}}{B_{\mathrm{cr}}}\right)^{2} E \\
& \simeq-1.3 \times 10^{-21} B_{\mathrm{mG}}^{2}\left(\frac{E}{\mathrm{TeV}}\right) \mathrm{cm}^{-1}, \\
\Delta_{B} & =\frac{g_{\gamma a} B_{\mathrm{t}}}{2} \simeq 1.7 \times 10^{-21} \mathrm{~g}_{11} B_{\mathrm{mG} \mathrm{cm}} \mathrm{cm}^{-1},
\end{aligned}
$$

where $E$ is the photon energy and $B_{\mathrm{cr}} \equiv m_{e}^{2} / e \simeq 4.41 \times$ $10^{13} \mathrm{G}$ is the critical magnetic field strength. Since we are only interested in orders of magnitude, we neglect different photon polarization states whose vacuum CottonMouton term have slightly different pre-factors, and we have averaged over directions, $\left\langle B_{\mathrm{t}}^{2}\right\rangle \simeq B^{2} / 3$. Further, in Eq. (9) we have used the abbreviations $B_{\mathrm{mG}} \equiv\left(B_{\mathrm{t}} / \mathrm{mG}\right)$, $g_{11} \equiv g_{\gamma a} \times 10^{11} \mathrm{GeV}$, and $m_{\mu \mathrm{eV}} \equiv\left(m_{a} / \mu \mathrm{eV}\right)$.

Only terms to lowest non-trivial order in $B$ are taken into account in Eq. (9). The term to next-higher order in $B$ in $\Delta_{\mathrm{CM}}$ is suppressed by a factor $\simeq \chi^{2} \equiv$ $\left[\left(E / m_{e}\right)\left(B / B_{\mathrm{cr}}\right)\right]^{2}[5]$. This is negligible for energies $E \lesssim 2 \times 10^{16}(\mathrm{kG} / B) \mathrm{eV}$. Furthermore, the magnetic field influences the phase space distribution of the electrons which modifies the plasma term to $\simeq(E / 2) \omega_{\mathrm{pl}}^{2} /\left(E^{2}-\right.$ $\left.\omega_{\mathrm{c}}^{2}\right)$ [5], where $\omega_{\mathrm{c}}=e B / m_{e}=\left(B / B_{\mathrm{cr}}\right) m_{e}$ is the electron cyclotron frequency in a non-relativistic plasma. The latter is thus negligible for energies $E \gtrsim \omega_{\mathrm{c}} \simeq$ $10^{-5}(B / \mathrm{kG}) \mathrm{eV}$. Furthermore, inelastic processes such as pair production and photon splitting in the magnetic field are suppressed as $\exp (-1 / \chi)$ and thus negligible for $\chi \ll 1[\underline{6}]$. Since we are mostly concerned with magnetic fields $B \lesssim 10^{4} \mathrm{G}$ and photon energies $E \lesssim \mathrm{PeV}$, both inelastic processes and higher order corrections to Eq. (9) can be neglected.

For propagation over $N$ coherence domains the total conversion probability of photons into axions can be derived as [3]

$$
P_{\gamma \rightarrow a} \simeq \frac{1}{3}\left[1-\exp \left(-3 N P_{0} / 2\right)\right],
$$

which saturates to $P_{\gamma \rightarrow a} \simeq \frac{1}{3}$ for $N P_{0} \gg 1$.

\section{A. Resonances}

In the following, let us denote the coherence length of the plasma and magnetic field by $\lambda$ with $\lambda \equiv \lambda /$ pc. The coherence length is of course always smaller than the size of the system.

MSW type resonances can occur in Eq. (9) when $\Delta_{\mathrm{pl}}$ becomes comparable to $\Delta_{a}$ or the modulus of $\Delta_{\mathrm{CM}}$ and the other contribution is negligible. In order for the resonance to lead to efficient conversion, it has to be adiabatic, requiring $2 \pi\left|\Delta_{\mathrm{pl}}^{\prime}+\Delta_{\mathrm{CM}}^{\prime}\right| \lesssim \Delta_{B}^{2}$ at the resonance, where the prime denotes the derivative with respect to 
the distance along the propagation direction. In the following we will estimate this derivative by the coherence length, i.e. ' $\rightarrow 1 / \lambda$.

One has $\Delta_{a}=\Delta_{\mathrm{pl}}$ at the electron density

$$
n_{e} \sim 7.1 \times 10^{8} \mathrm{~m}_{\mu \mathrm{eV}}^{2} \mathrm{~cm}^{-3} .
$$

This leads to an adiabatic resonance at energies satisfying

$$
\begin{aligned}
& E \gtrsim 20 g_{11}^{-2} m_{\mu \mathrm{eV}}^{2} \lambda_{\mathrm{pc}}^{-1} B_{\mathrm{mG}}^{-2} \mathrm{TeV}, \\
& E \gtrsim 4.4 m_{\mu \mathrm{eV}} B_{\mathrm{mG}}^{-1} \mathrm{TeV} .
\end{aligned}
$$

In Eq. (12), the first condition results from the adiabaticity requirement with $\Delta_{\mathrm{osc}}^{\prime} \sim \Delta_{\mathrm{pl}} / \lambda$ and the second from $\Delta_{\mathrm{CM}} \lesssim \Delta_{a}$. As a result, strong $\gamma$-ray flux suppression is expected at energies satisfying Eq. (12).

One has $\Delta_{\mathrm{CM}}=-\Delta_{\mathrm{pl}}$ at the energy

$$
E \sim 5.2\left(\frac{n_{e}}{10^{3} \mathrm{~cm}^{-3}}\right)^{1 / 2} B_{\mathrm{mG}}^{-1} \mathrm{GeV},
$$

This leads to an adiabatic resonance provided that at this energy $\Delta_{a} \lesssim\left|\Delta_{\mathrm{pl}}\right|$ and that the adiabaticity condition is fulfilled with $\Delta_{\mathrm{osc}}^{\prime} \sim \Delta_{\mathrm{CM}} / \lambda$. This translates into

$$
\begin{aligned}
n_{e} & \gtrsim 7.1 \times 10^{8} m_{\mu \mathrm{eV}}^{2} \mathrm{~cm}^{-3}, \\
E & \lesssim 0.95 g_{11}^{2} \lambda_{\mathrm{pc}} \mathrm{TeV},
\end{aligned}
$$

respectively. Note that the first condition in Eq. (14) together with Eq. (13) implies $E \gtrsim 4.4 m_{\mu \mathrm{eV}} B_{\mathrm{mG}}^{-1} \mathrm{TeV}$, such that this type of resonance starts to be relevant at energies just above where the first type of resonance ceases. This is understood since for the first resonance we had $\left|\Delta_{\mathrm{CM}}\right| \lesssim \Delta_{a}$, whereas for the second $\Delta_{a} \lesssim \Delta_{\mathrm{pl}}=$ $\left|\Delta_{\mathrm{CM}}\right|$.

Eq. (12) and the second inequality in Eq. (14) combined thus lead to resonances in the energy range

$$
\begin{aligned}
E & \gtrsim 20 g_{11}^{-2} m_{\mu \mathrm{eV}}^{2} \lambda_{\mathrm{pc}}^{-1} B_{\mathrm{mG}}^{-2} \mathrm{TeV}, \\
E & \lesssim 0.95 g_{11}^{2} \lambda_{\mathrm{pc}} \mathrm{TeV},
\end{aligned}
$$

provided the following conditions are satisfied:

$$
\begin{aligned}
g_{11} & \gtrsim 2.1\left(\frac{m_{\mu \mathrm{eV}}}{\lambda_{\mathrm{pc}} B_{\mathrm{mG}}}\right)^{1 / 2}, \\
n_{e} & \gtrsim 7.1 \times 10^{8} \mathrm{~m}_{\mu \mathrm{eV}}^{2} \mathrm{~cm}^{-3} .
\end{aligned}
$$

Here the first condition comes from the requirement that Eq. (15) represents a finite energy range, and the second condition results from Eq. (11) and the first inequality in Eq. (14). For a given coupling $g_{11}$ resonances occur only for coherence lengths (and thus system sizes) satisfying

$$
d \gtrsim \lambda \gtrsim 1.4 \times 10^{19} g_{11}^{-2} m_{\mu \mathrm{eV}} B_{\mathrm{mG}}^{-1} \mathrm{~cm} .
$$

Inserting Eq. (17) into Eq. (15) thus also leads to the inequalities

$$
E_{\mathrm{min}}^{\mathrm{r}} \lesssim 4.4 m_{\mu \mathrm{eV}} B_{\mathrm{mG}}^{-1} \mathrm{TeV} \lesssim E_{\max }^{\mathrm{r}} .
$$

for the minimal and maximal photon energy $E_{\min }^{\mathrm{r}}$ and $E_{\max }^{\mathrm{r}}$, respectively, at which resonances occur.

\section{B. Non-resonant Oscillations}

Let us denote the coherence length of the magnetic field by $\lambda$ and the propagation length with $d$, so that we have $N \sim d / \lambda \gtrsim 1$ domains. For $\Delta_{\text {osc }} \lambda \lesssim 1$, we have from Eq. (7) $P_{0} \sim\left(\Delta_{B} \lambda\right)^{2}$. In the opposite limit, $\Delta_{\text {osc }} \lambda \gtrsim 1$, we have $P_{0} \sim 2\left(\Delta_{B} / \Delta_{\text {osc }}\right)^{2}$. Altogether, with Eq. (10) this yields for the photon survival probability $P_{\gamma \rightarrow \gamma} \equiv 1-P_{\gamma \rightarrow a}$,

$P_{\gamma \rightarrow \gamma} \sim \frac{1}{3}\left[2+\exp \left(-\frac{3}{2} \min \left[\Delta_{B}^{2} d \lambda, 2 N\left(\Delta_{B} / \Delta_{\text {osc }}\right)^{2}\right]\right)\right]$.

For given axion parameters, the spectra predicted within scenarios of $\gamma$-ray sources will be modified by this factor. Eq. (19) can only be of order unity and thus lead to observable effects, if both $\Delta_{B}^{2} d \lambda \gtrsim 1$ and $2\left(\Delta_{B} / \Delta_{\text {osc }}\right)^{2} N \gtrsim$ 1. With Eq. (9), the first condition yields

$$
(d \lambda)^{1 / 2} \gtrsim 5.7 \times 10^{19} g_{11}^{-1} B_{\mathrm{mG}}^{-1} \mathrm{~cm} .
$$

This results in the two conditions

$$
d \gtrsim 5.7 \times 10^{19} g_{11}^{-1} B_{\mathrm{mG}}^{-1} \mathrm{~cm},
$$

and

$$
1 \leq N^{1 / 2} \equiv\left(\frac{d}{\lambda}\right)^{1 / 2} \lesssim 0.054 d_{\mathrm{pc}} g_{11} B_{\mathrm{mG}},
$$

or equivalently

$$
\lambda=\frac{d}{N} \gtrsim 320 g_{11}^{-2} d_{\mathrm{pc}}^{-1} B_{\mathrm{mG}}^{-2} \mathrm{pc},
$$

where $d_{\mathrm{pc}} \equiv(d / \mathrm{pc})$.

The second condition from Eq. (19) leads to the three separate inequalities $\Delta_{B} \gtrsim \Delta_{\mathrm{a}} N^{-1 / 2}, \Delta_{B} \gtrsim$ $\left|\Delta_{\mathrm{pl}}\right| N^{-1 / 2}$, and $\Delta_{B} \gtrsim \Delta_{\mathrm{CM}} N^{-1 / 2}$. Substituting Eq. (9) results in

$$
\begin{aligned}
& E \gtrsim 15 g_{11}^{-1} m_{\mu \mathrm{eV}}^{2} N^{-1 / 2} B_{\mathrm{mG}}^{-1} \mathrm{TeV} \\
& E \gtrsim 21\left(\frac{n_{e}}{10^{3} \mathrm{~cm}^{-3}}\right) g_{11}^{-1} N^{-1 / 2} B_{\mathrm{mG}}^{-1} \mathrm{MeV}, \\
& E \lesssim 1.3 g_{11} N^{1 / 2} B_{\mathrm{mG}}^{-1} \mathrm{TeV}
\end{aligned}
$$

Note that the scaling of these energies with $\lambda$ are different from the scaling of the resonance energies Eq. (15): Large coherence lengths, or small $N$, favor a broad resonance energy range, whereas small coherence lengths will tend to lead to non-resonant transitions, as long as Eq. (22) is satisfied. The absolute minimal and maximal photon energies at which non-resonant oscillations occur are given by setting $N=1$ in Eq. (24).

We can now eliminate $N$ or equivalently $\lambda$ from Eq. (24) by using Eq. (21):

$$
\begin{aligned}
E & \gtrsim 278 g_{11}^{-2} m_{\mu \mathrm{eV}}^{2} d_{\mathrm{pc}}^{-1} B_{\mathrm{mG}}^{-2} \mathrm{TeV} \\
E & \gtrsim 389\left(\frac{n_{e}}{10^{3} \mathrm{~cm}^{-3}}\right) g_{11}^{-2} d_{\mathrm{pc}}^{-1} B_{\mathrm{mG}}^{-2} \mathrm{MeV}, \\
E & \lesssim 0.07 g_{11}^{2} d_{\mathrm{pc}} \mathrm{TeV}
\end{aligned}
$$


For Eqs. (24) and (25) to be satisfied for a finite energy range the following conditions are implied:

$g_{11} \gtrsim 3.4 m_{\mu \mathrm{eV}} N^{-1 / 2} \gtrsim 0.13\left(\frac{m_{\mu \mathrm{eV}}}{d_{\mathrm{pc}} B_{\mathrm{mG}}}\right)^{1 / 2}$,

$n_{e} \lesssim 6.2 \times 10^{7} g_{11}^{2} \mathrm{Ncm}^{-3} \lesssim 1.8 \times 10^{5} g_{11}^{4} d_{\mathrm{pc}}^{2} B_{\mathrm{mG}}^{2} \mathrm{~cm}^{-3}$

where in the second expressions we have substituted Eq. (22). The condition on $g_{11}$ is very similar to the resonance condition Eq. (16).

Coupling and mass of the QCD axion roughly satisfy $g_{11} \sim 10^{-5} m_{\mu \mathrm{eV}}$, and thus experimental limits imply $m_{a} \lesssim 1 \mathrm{eV}$. The first condition in Eq. (26) then implies

$$
\begin{aligned}
N & \gtrsim 1.2 \times 10^{11}, \\
d_{\mathrm{pc}} B_{\mathrm{mG}} & \gtrsim 18 g_{11}^{-1} \sim 1.8 \times 10^{6} \mathrm{~m}_{\mu \mathrm{eV}}^{-1},
\end{aligned}
$$

where in the second line we have used Eq. (21). In 2005, the PVLAS [7] experiment has seen indications for axionphoton mixing with

$$
\begin{aligned}
m_{a} & \simeq 1.3 \mathrm{meV} \\
g_{\gamma a} & \simeq 3 \times 10^{-6} \mathrm{GeV}^{-1}
\end{aligned}
$$

which since recently, however, is considered to probably have been an experimental effect [8]. In any case, the "PVLAS axion" would fulfill the first condition in Eq. (26).

\section{Other Conditions}

A further condition comes from the requirement that the length scale over which the emission is created cannot be larger than the variability time scale of the source. The size of the emission region is determined by the length scale over which the plasma becomes transparent to $\gamma \gamma$ pair production. Emissions of active and radio galaxies often vary on times scales of days or even hours [9, 10], corresponding to scales $\lesssim 10^{14-15} \mathrm{~cm}$. If these are the same length scales over which significant photon-axion conversion occurs, then one also has the condition $\lambda \leq d \lesssim 10^{14-15} \mathrm{~cm}$, except in case of relativistic beaming.

\section{PROSPECTS FOR SPECIFIC SOURCES}

A lower limit on the source magnetic field times the propagation length can actually be obtained by requiring that it accelerates cosmic rays of charge $Z e$ up to the maximal energy $E_{\text {cr }}$ observed from that source:

$$
d \gtrsim \frac{E_{\mathrm{cr}}}{Z e B} \simeq 3.3 \times 10^{12} Z^{-1}\left(\frac{E_{\mathrm{cr}}}{\mathrm{TeV}}\right) B_{\mathrm{mG}}^{-1} \mathrm{~cm} .
$$

This implies that sources that accelerate particles up to

$$
E_{\text {cr }} \gtrsim 1.7 \times 10^{19} Z g_{11}^{-1} \mathrm{eV}
$$

fulfill the condition Eq. (21) independently of the magnetic field strength. The coherence length of the fields depends on the acceleration model and has to satisfy the additional condition Eq. 23). Sources of ultra-high energy cosmic rays with energies $E_{\text {cr }}>10^{18} \mathrm{eV}$ are thus promising objects that may exhibit axion-photon mixing induced modifications of their photon spectra for couplings $g_{11} \gtrsim 1.7\left(Z 10^{19} \mathrm{eV} / E_{\mathrm{cr}}\right)$.

Whereas this condition for the occurrence of photonaxion oscillations does not depend on size or magnetic field strength of the accelerator, the energies at which such oscillations could modify photon spectra do depend on these parameters: Eqs. (15) and (25) show that the minimal and maximal energies at which significant oscillation effects can occur, are given by

$$
\begin{aligned}
E_{\mathrm{min}} & \sim 19 g_{11}^{-2} m_{\mu \mathrm{eV}}^{2} B_{\mathrm{mG}}^{-1}\left(\frac{Z 10^{18} \mathrm{eV}}{E_{\mathrm{cr}}}\right) \mathrm{TeV} \\
& \sim 18 g_{11}^{-2} m_{\mu \mathrm{eV}}^{2} d_{\mathrm{pc}}\left(\frac{Z 10^{18} \mathrm{eV}}{E_{\mathrm{cr}}}\right)^{2} \mathrm{TeV} \\
E_{\max } & \sim 0.95 g_{11}^{2} d_{\mathrm{pc}} \mathrm{TeV},
\end{aligned}
$$

where we have used the relation Eq. (29).

Among the extragalactic objects which have been seen in very high energy $\gamma$-rays and where photon-axion conversion could occur are Markarian 421 11], Markarian 501 [11, 12], the blazar 1ES 1101-232 [13], and the variable core 9] and flaring knots [14] of M87. Markarian 421 is also variable in $\gamma$-rays[10]. Recent reviews on leptonic and hadronic models of blazar emission can be found in Refs. [15, 16]. The spectrum of a typical AGN has a double-peaked power spectrum. In leptonic models, the high energy peak is caused by inverse Compton scattering of accelerated electrons on the ambient photon field, whereas the low energy peak is due to synchrotron emission of these same electrons. The energies and relative power flux in these peaks thus contains information on the magnetic fields in the emission region. The estimated magnetic field strengths range between $\sim 10 \mathrm{mG}$ and $\sim 10 \mathrm{G}$ over scales, depending on the models used, typically of order $d \gtrsim 10^{-3} \mathrm{pc}$. This would also suggest possible cosmic ray acceleration up to $\sim 10^{18} \mathrm{eV}$. For axion parameters $g_{11} \sim 1, m_{\mu \mathrm{eV}} \sim 1$, Eq. (31) then suggests photon-axion oscillation effects at $\mathrm{GeV}$ energies, consistent with the findings of Ref. [17].

For the galactic center fields of order $10 \mathrm{G}$ and up to $10^{4} \mathrm{G}$ have been discussed in Ref. [18, 19]. The galactic center is sometimes thought to accelerate cosmic rays up to $\sim 10^{18} \mathrm{eV}[20]$. Eq. (31) would then imply significant non-resonant axion-photon oscillations down to $\mathrm{MeV}$ energies.

\section{A. Former Work}

The original indications from PVLAS for axion-like particles with rather strong couplings, Eq. (28), although in contradiction with present astrophysical constraints, 
has motivated the study of several possible astrophysical effects. Ref. [21, 22], considered variable $\gamma$-ray light curves from double pulsars as signatures for the PVLAS axion-photon mixing parameters. In this scenario, a surface pulsar magnetic field $B \sim 10^{12} \mathrm{G}$ yields $B \sim 10^{4} \mathrm{G}$, $N \sim 1$ in the accretion region between the pulsars where the $\gamma$-rays are produced, and the Goldreich-Julian plasma density is $n_{e} \sim 10^{3} \mathrm{~cm}^{-3}$. Eq. (24) implies a modulation for $\mathrm{MeV} \lesssim E \lesssim 100 \mathrm{GeV}$, as obtained in Ref. [21, 22]. Note that for less coherent fields between the two compact stars, $N>1$, the modulation would extend down to lower energies.

Ref. [4] considered the modification of the galactic center $\gamma$-ray flux by PVLAS axions, for $B \sim \mu \mathrm{G}, \lambda \sim$ $0.01 \mathrm{pc}, d \sim 10 \mathrm{kpc}$, thus $N \sim 10^{6}$. Thus Eq. (24) results in a modification at energies $10 \mathrm{TeV} \lesssim E \lesssim 10^{12} \mathrm{TeV}$. Note that the coherence length of the Galactic magnetic field is not very well known [23]. For $\lambda \gtrsim$ pc, the effect would disappear for all observable energies $E \lesssim 100 \mathrm{TeV}$. Measurement of Faraday rotation from pulsar pairs suggest that the cell size of the random component of the galactic magnetic field may indeed be of order $50 \mathrm{pc}$ [24, 25].

No resonances are expected in these two scenarios due to the second condition in Eq. (16), unless $m \lesssim 10^{-8} \mathrm{eV}$.

Ref. [26] constrained axion-photon mixing by using quasar spectra mostly in the optical, considering mixing in intergalactic fields of strength $B \lesssim 10^{-9} \mathrm{G}$ only. In this case such spectra are only sensitive to axion masses $m_{a} \lesssim 10^{-15} \mathrm{eV}$, consistent with Eqs. (15) and (25). For larger axion masses $m \sim 10^{-10} \mathrm{eV}$, such conversions in intergalactic magnetic fields would show up at $\mathrm{TeV}$ energies, as discussed in Ref. [27].

Ref. 17] discussed the modification of $\gamma$-ray spectra from AGNs by axion-photon mixing, using magnetic fields and length scales according to the Hillas criterion for accelerating particles to the pertinent energies. However, they did not consider resonances.

Note that emission from the surface of a neutron star or magnetar with $d \sim 10^{6} \mathrm{~cm}, B \sim 10^{12}-10^{15} \mathrm{G}$, $n_{e} \sim 10^{34} \mathrm{~cm}^{-3}$ according to the second condition in Eq. (26) could not give significant non-resonant transitions for $g_{11} \lesssim 10^{5}$. Furthermore, according to Eq. (15), resonances can only occur at very low energies $E \lesssim$ $0.3 g_{11}^{2} \mathrm{eV}$.

\section{B. The central engine of AGNs}

Black hole accretion launches jets by magnetohydrodynamical processes. For spherical Bondi-accretion, the accretion rate is $\dot{M} \sim 4 \pi r^{2} n_{e} m_{p} \beta$ where $m_{p}$ is the proton mass and $\beta \sim \beta_{0}\left(r_{\mathrm{S}} / r\right)^{1 / 2}$ are the density and velocity of the accretion flow, respectively. Here, $\beta_{0} \sim 0.1$ and $r_{\mathrm{S}}=2 G_{\mathrm{N}} M \simeq 2.96 \times 10^{14} M_{9} \mathrm{~cm}$ is the Schwarzschild radius for a black hole of mass $M=M_{9} M_{\odot}$. Introducing the Eddington luminosity, $L_{\mathrm{Edd}}(M) \simeq 1.3 \times$ $10^{47} M_{9} \mathrm{erg} \mathrm{s}^{-1}$, the accretion rate can be written as
$\dot{M}=f_{\mathrm{Edd}} L_{\mathrm{Edd}}(M) / \eta$, where $\eta$ is the efficiency with which accretion is converted into electromagnetic radiation, and $f_{\mathrm{Edd}}=L_{\mathrm{bol}} / L_{\mathrm{Edd}}(M)$ is the ratio of the bolometric luminosity to the Eddington luminosity. AGNs have duty cycles of a few percent and in their active periods have $\eta \sim f_{\text {Edd }} \sim 0.1$.

From this we can estimate the plasma density in the accretion flow by $n_{e} \sim \dot{M} /\left(4 \pi r^{2} m_{p} \beta\right)$ which close to the Schwarzschild radius yields

$$
n_{e} \sim 2.6 \times 10^{9}\left(\frac{f_{\mathrm{Edd}}}{\eta \beta_{0}}\right) M_{9}^{-1}\left(\frac{r}{r_{\mathrm{S}}}\right)^{-3 / 2} \mathrm{~cm}^{-3} .
$$

If the magnetic fields are roughly in equipartition with the accretion flow at radius $r$, one has $B^{2} /(8 \pi) \sim$ $n_{e} m_{p} \beta^{2}$. This results in [28]

$$
\begin{aligned}
B & \sim 3.9 \beta_{0}^{1 / 2}\left(\frac{\dot{M}}{M_{\odot} \mathrm{yr}^{-1}}\right)^{1 / 2} M_{9}^{-1}\left(\frac{r}{r_{\mathrm{S}}}\right)^{-5 / 4} \mathrm{kG} \\
& =5.9\left(\frac{\beta_{0} f_{\mathrm{Edd}}}{\eta}\right)^{1 / 2} M_{9}^{-1 / 2}\left(\frac{r}{r_{\mathrm{S}}}\right)^{-5 / 4} \mathrm{kG} .
\end{aligned}
$$

where in the second expression the accretion rate has been written in terms of the Eddington luminosity. Note that these are very rough order of magnitude estimates which, however, will be sufficient for our purpose.

If accretion is non-spherical, but instead occurs in a disk, as is in general the case for very luminous AGNs, for a given accretion rate, the plasma density and magnetic field strengths tend to be larger than the estimates Eqs. (32) and (33). In these and all subsequent formulas, this is essentially mimicked by an electromagnetic efficiency $\eta$ smaller by a factor of roughly the height of the disk relative to its radius. This will be very roughly the Lorentz factor of the resulting jet. The geometry is, however, more complicated in this case and photon-axion oscillations may be suppressed perpendicular to the disk. The strongest effects may then only occur in directions of the plane of the disk. As we will see below, both the minimal Eddington ratio $f_{\text {Edd }}$ and the energy for which oscillation effects occur are proportional to $\eta$, whereas other parameters are largely insensitive to $\eta$.

In any case, more detailed theoretical models of magnetized accretion disks lead to magnetic fields of order $10^{4} \mathrm{G}$ in the disk and $\sim 10^{2} \mathrm{G}$ above the disk [29]. Such values are consistent with the rough estimates in Eq. (33). In addition, supermassive black holes may have a dipole magnetic field with a maximal field strength of $\sim 2 \times 10^{10} M_{9}^{-1} \mathrm{G}$ at the Schwarzschild radius [30].

But how coherent are these fields? We note that they may be made coherent by dynamo effects and/or magnetorotational instability (MRI) Jet launching by MHD effects may indeed require coherent, very non-thermal fields. In this case the fields would be coherent on roughly the Schwarzschild radius.

Magnetic fields of order $10 \mathrm{G}$ in the vicinity of the radio galaxy M87 have been discussed in Ref. [31]. This 
value is consistent with the estimate Eq. (33) if M87 is in a low accretion state, $f_{\mathrm{Edd}} \sim 10^{-6}$, which is the case according to present measurements and theories 31].

The variability constraint is fulfilled automatically since AGNs cannot be variable on time scales smaller than the Schwarzschild radius.

\section{Resonances}

Comparing the second condition in Eq. (16) with Eq. (32) implies the condition

$$
M \lesssim 3.7 \times 10^{9} m_{\mu \mathrm{eV}}^{2}\left(\frac{f_{\mathrm{Edd}}}{\eta \beta_{0}}\right) M_{\odot}
$$

for a resonance to occur above the Schwarzschild radius.

Using Eq. (33) and assuming $\lambda \propto r$, Eq. (15) shows that the widest energy range is achieved for the smallest radii. Further, the first condition in Eq. (16) implies the least stringent condition on $g_{11}$ for the smallest radii. We thus get

$$
g_{11} \gtrsim 8.8 \times 10^{-2} N^{1 / 2} m_{\mu \mathrm{eV}}^{1 / 2} M_{9}^{-1 / 4}\left(\frac{\eta}{\beta_{0} f_{\mathrm{Edd}}}\right)^{1 / 4} .
$$

Thus, when the above conditions Eq. (34) and Eq. (35) are fulfilled, a strong $\gamma$-ray flux suppression occurs for an energy interval given by substituting the field strength Eq. (33) close to the Schwarzschild radius into Eq. (15),

$$
\begin{aligned}
E & \gtrsim 6 \times 10^{3} g_{11}^{-2} m_{\mu \mathrm{eV}}^{2} N\left(\frac{\eta}{\beta_{0} f_{\mathrm{Edd}}}\right) \mathrm{eV}, \\
E & \lesssim 90 g_{11}^{2} N^{-1} M_{9} \mathrm{MeV},
\end{aligned}
$$

where $N \equiv \lambda / r_{\mathrm{S}}$ is the number of coherent domains within a Schwarzschild range. Note that the lower limit does not depend on the black hole mass. In Fig. 1 we show the energy range Eq. (36) for fields coherent over the Schwarzschild radius, $N=1$, for an AGN of the type of Mrk421 or Mrk501, with $M \sim 10^{6} M_{\odot}, f_{\text {Edd }} \sim 10^{-3}$. For couplings approaching the experimental upper limit, $g_{a \gamma} \lesssim 10^{-10} \mathrm{GeV}^{-1}$, resonances can occur down to energies $\sim 600(m / 0.1 \mu \mathrm{eV})^{2}\left(f_{\text {Edd }} / 10^{-3}\right)^{-1} \mathrm{eV}$ which can extend into the $\mathrm{keV}$ regime.

The condition on the coherence scale Eq. (17) reads

$$
\begin{aligned}
& f_{\mathrm{Edd}} \gtrsim 6.1 \times 10^{-5}\left(\frac{\eta}{\beta_{0}}\right) g_{11}^{-4} m_{\mu \mathrm{eV}}^{2} M_{9}^{-1}, \\
& N^{-1} \equiv \frac{\lambda}{r_{\mathrm{S}}} \gtrsim 7.8 \times 10^{-3} g_{11}^{-2} m_{\mu \mathrm{eV}} M_{9}^{-1 / 2}\left(\frac{\eta}{\beta_{0} f_{\mathrm{Edd}}}\right)^{1 / 2},
\end{aligned}
$$

where the first condition follows from the second observing that $N \geq 1$.

\section{Non-resonant Oscillations}

Substituting $d=r=r_{\mathrm{S}}$ in the conditions Eq. (21) and Eq. (22) and in Eq. (33) results in requirements similar

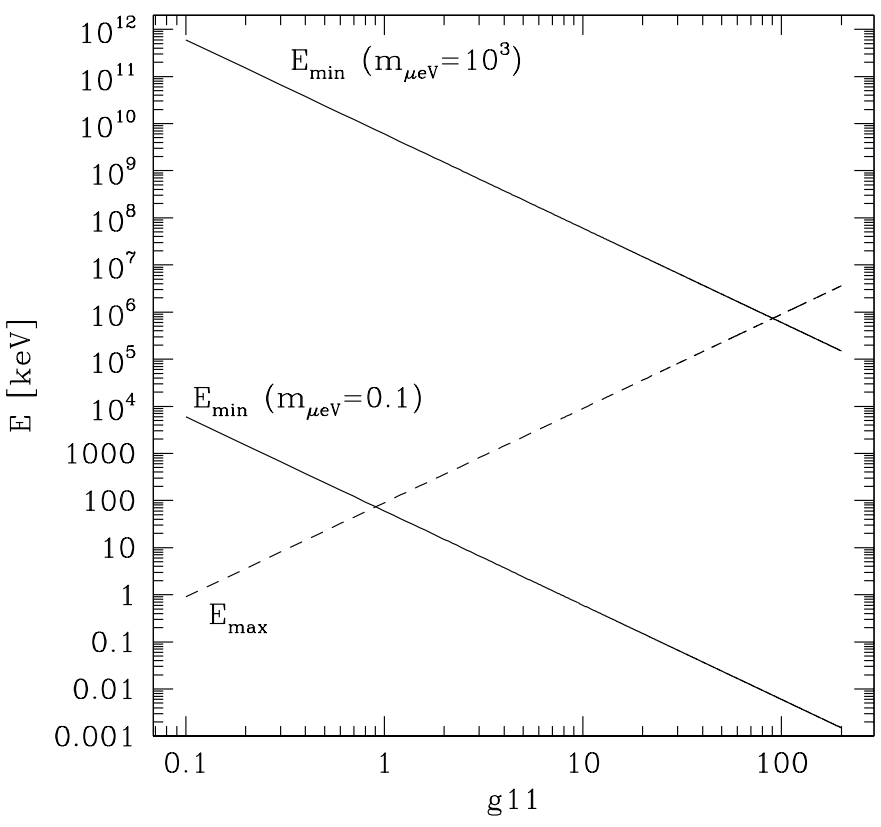

FIG. 1: Maximal and minimal energies Eq. (36) for which axion-photon resonances occur, for $M \sim 10^{6} M_{\odot}, f_{\mathrm{Edd}} \sim$ $10^{-3}, \beta_{0}=\eta=0.1, \lambda=r_{\mathrm{S}}$. Whenever $E_{\min } \leq E_{\max }$, adiabatic resonances occur. Note that in this case also the conditions Eqs. (34), (35), and (37) are fulfilled.

to Eq. (37),

$$
\begin{aligned}
& f_{\mathrm{Edd}} \gtrsim 1.1 \times 10^{-3}\left(\frac{\eta}{\beta_{0}}\right) g_{11}^{-2} M_{9}^{-1}, \\
& N^{-1} \equiv \frac{\lambda}{r_{\mathrm{S}}} \gtrsim 1.1 \times 10^{-3} g_{11}^{-2} M_{9}^{-1} \frac{\eta}{\beta_{0} f_{\mathrm{Edd}}} .
\end{aligned}
$$

We remark that the first constraint is easily fulfilled for luminous AGNs which have $f_{\mathrm{Edd}} \gtrsim 10^{-2}$, especially for relatively large axion-photon coupling. The central black hole of our Galaxy has $f_{\text {Edd }} \sim 10^{-8}$.

Note that the condition on the coherence length in Eq. (38) tends to be less stringent than Eq. (37), consistent with the fact that more coherent fields tend to lead to resonances.

Using the plasma density Eq. (32) and again substituting $d=r=r_{\mathrm{S}}$ and Eq. (33), the two conditions in Eq. (26) translate into

$$
\begin{aligned}
& g_{11} \gtrsim 5.5 \times 10^{-3} m_{\mu \mathrm{eV}}^{1 / 2} M_{9}^{-1 / 4}\left(\frac{\eta}{\beta_{0} f_{\mathrm{Edd}}}\right)^{1 / 4}, \\
& g_{11} \gtrsim 0.46 \beta_{0}^{-1 / 2} M_{9}^{-1 / 2} .
\end{aligned}
$$

This implies that emission from massive black holes can be sensitive to rather small coupling constants. The range of coupling constants given by Eq. (39) is shown in Fig. 2 ,

Thus, when the above conditions Eqs. (38) and Eqs. (39) are fulfilled, a $\gamma$-ray flux suppression by about 


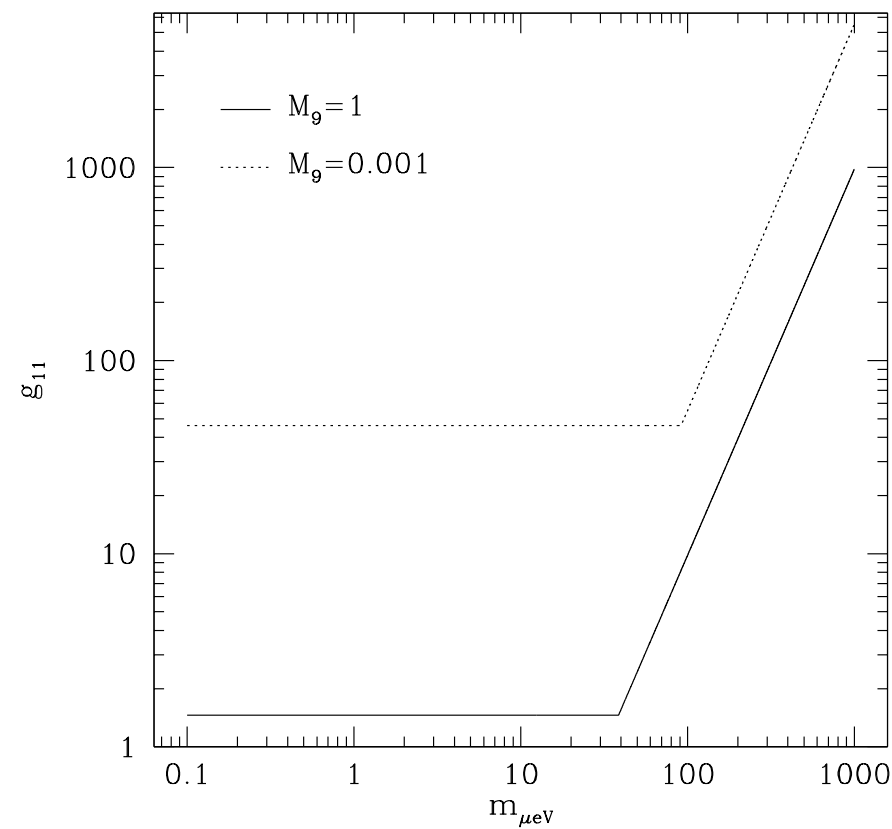

FIG. 2: Range of coupling constants and axion masses Eq. (39) for which non-resonant axion-photon oscillations can influence the $\gamma$-ray spectra from AGNs with $f_{\text {Edd }}=10^{-3}$.

a factor $\simeq 2 / 3$ occurs for energies

$$
\begin{aligned}
E \gtrsim & 2.5 g_{11}^{-1} m_{\mu \mathrm{eV}}^{2} N^{-1 / 2}\left(\frac{\eta}{\beta_{0} f_{\mathrm{Edd}}}\right)^{1 / 2} M_{9}^{1 / 2} \mathrm{MeV} \\
& \gtrsim 0.083 g_{11}^{-2} m_{\mu \mathrm{eV}}^{2}\left(\frac{\eta}{\beta_{0} f_{\mathrm{Edd}}}\right) \mathrm{MeV} \\
E \lesssim & 0.22 g_{11}^{-1} N^{1 / 2}\left(\frac{\eta}{\beta_{0} f_{\mathrm{Edd}}}\right)^{1 / 2} M_{9}^{1 / 2} \mathrm{MeV} \\
& \lesssim 6.6 M_{9} \mathrm{MeV}
\end{aligned}
$$

where for the second expressions we have used the constraint on the number of domains in the second line of Eq. (38). The third condition that can be obtained from Eq. (24) is less stringent than the one given.

Note that the energies Eq. (40) tend to be in the hard $\mathrm{X}$-ray to soft $\gamma$-ray range except for very strong couplings of the PVLAS type for which these energies can extend down to the optical range. Such photons are not absorbed by pair production and there is plenty of data at such energies that could be searched for the spectral features discussed here.

\section{AGN jets and hot-spots}

AGN jets and hot-spots seem to be likely candidates to fulfill the requirements for observable photon-axion conversion, as the condition Eq. (21) is satisfied for all couplings $g_{11} \gtrsim 10^{-2}$ in such environments since even the transverse dimension of the jets are of order kpc and fields are at least of order $100 \mu \mathrm{G}$ 32, 33, 34]. The first condition in Eq. (26) shows that this allows significant effects down to couplings $g_{11} \gtrsim 0.013 \mathrm{~m}_{\mu \mathrm{eV}}^{1 / 2}$, provided the coherence scale $\lambda \sim$ pc. Furthermore, the plasma density in jets is assumed as $n_{e} \lesssim 10^{5} \mathrm{~cm}^{-3}$, about a factor 100 higher than the ambient density of an average galaxy, as expected for termination shocks. Thus, the second condition in Eq. (26) is also satisfied, unless $g_{11} \lesssim 0.1$. According to Eq. (25), the resulting features would show up at $\mathrm{TeV}$ energies. However, due to Eq. (14), resonances would only occur for $m_{a} \lesssim 1.2 \times 10^{-8} \mathrm{eV}$.

In the proton synchrotron model, field strengths of up to $10 \mathrm{mG}$ have been discussed in Ref. [35]. For axions with $g_{11} \sim 1, m_{a} \sim \mu \mathrm{eV}$ Eqs. (24) and (25) thus imply possible effects down to $\sim \mathrm{GeV}$ energies, if $\lambda \sim 0.1 \mathrm{pc}$.

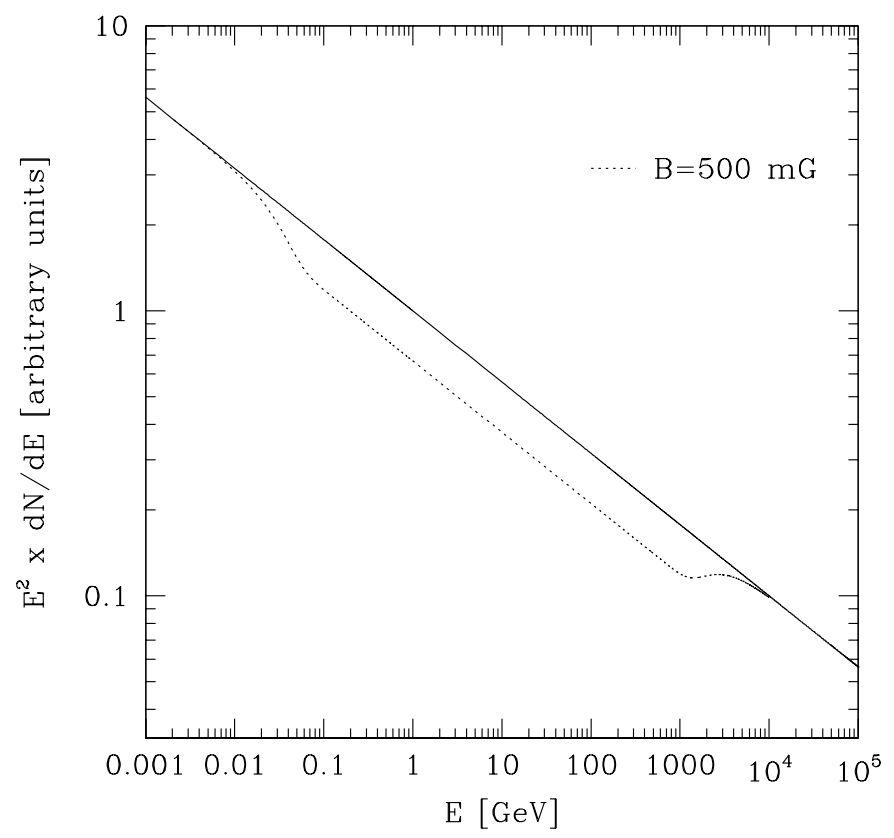

FIG. 3: Modification of the $\gamma$-ray spectrum of an AGN of mass $M=10^{9} M_{\odot}$, for $B=0.5 \mathrm{G}$ over $2 \times 10^{6}$ Schwarzschild radii, or $\sim 200 \mathrm{pc}$ with coherence length $\lambda$ equal to 10 times the Schwarzschild radius, or $\sim 10^{-3}$ pc. The assumed injection spectrum is $\propto E^{-2.25}$. The axion parameters are $g_{\gamma a}=10^{-11} \mathrm{GeV}^{-1}, m_{a}=1 \mu \mathrm{eV}$. The modification factor $P_{\gamma \rightarrow \gamma}$ is given by Eq. (19). Spectral modification by pair production has not been taken into account and is negligible for redshifts $z \lesssim 0.03[36]$.

As an example for the effect of non-resonant oscillations we show in Fig. 3 the modification of the $\gamma$-ray spectrum of a quasar around the maximal energy given by Eq. (24). The parameters used are typical for AGNs with central black hole masses around $10^{9} M_{\odot}$. The magnetic field is assumed of order a Gauss over length scales $10^{6}$ times the Schwarzschild radius. Such fields can occur in models of kpc scale jets which emit $\mathrm{MeV} \gamma$-rays produced by inverse Compton scattering of accelerated electrons on low energy synchrotron photons and external photons [16, 32], and in ultra-compact jets 37]. However, variability over scales of months or less would imply 
that the length scales over which significant photon-axion conversion occurs would be much larger than the size of the region where most of the emission is produced. This region would have to be close to the black hole, except if the jet is oriented toward the observer such that relativistic beaming compresses the time scales.

\section{CONCLUSIONS}

We have investigated the possibilities for successful photon-axion conversion in $\gamma$-ray sources such as the discs and jets of AGNs. We have derived conditions for resonant and non-resonant oscillations and find that a significant conversion is possible for standard axion parameters with coupling $g_{11} \sim 1$ and axion mass $m_{\mu \mathrm{eV}} \sim 1$, which are allowed by present experimental and astrophysical constraints. Of course the efficiency of conversion depends on the strength of the magnetic field in the vicinity of the black hole. Values between $\sim 0.01 \mathrm{G}$ and several $\mathrm{G}$ are under discussion. Resonant effects, leading to gaps in the observed spectrum, are observable for plasma densities $n_{e} \gtrsim 7.1 \times 10^{8} \mathrm{~m}_{\mu \mathrm{eV}}^{2} \mathrm{~cm}^{-3}$ and energies between $\sim$ $\mathrm{keV}$ and $\mathrm{MeV}$ for emissions from the central engines of active galactic nuclei. Such resonances can occur between the axion mass term and the plasma frequency term as well as between the plasma frequency term and the vac- uum Cotton-Mouton shift. Assuming a limited region of production and conversion of order the gravitational radius we find that non-resonant effects can occur again in the $\mathrm{keV}$ and $\mathrm{MeV}$ energy range, provided that also the magnetic field is coherent over this distance. AGN jets and hot-spots should provide an even more interesting site with field strength of order $\mu \mathrm{G}-\mathrm{mG}$ and jet sizes of order kpc. In these scenarios we find that a significant conversion is possible for $\mathrm{MeV}-\mathrm{TeV}$ energies. Our findings should be testable in high energy $\gamma$-ray experiments. Additionally, though all our considerations encompassed normal axion parameters, our limits can be applied to axion-like particles like the PVLAS axion. In this case effects should even be observable down to the optical scale and thus be already testable.

\section{Acknowledgments}

We thank G. Raffelt for useful comments and discussions. This work was partly supported by the Deutsche Forschungsgemeinschaft under the grant TR-27 "Neutrinos and beyond", the cluster of excellence for fundamental physics: Origin and Structure of the Universe, and by the European Union under the ILIAS project, contract No. RII3-CT-2004-506222. KAH would like to thank the APC for hospitality.
[1] R. Battesti et al., "Axion searches in the past, at present, and in the near future," arXiv:0705.0615 [hep-ex].

[2] G. Raffelt and L. Stodolsky, "Mixing of the Photon with Low Mass Particles," Phys. Rev. D 37, 1237 (1988).

[3] A. Mirizzi, G. G. Raffelt and P. D. Serpico, "Photon axion conversion in intergalactic magnetic fields and cosmological consequences," arXiv:astro-ph/0607415

[4] A. Mirizzi, G. G. Raffelt and P. D. Serpico, "Signatures of axion-like particles in the spectra of $\mathrm{TeV}$ gamma-ray sources," arXiv:0704.3044 [astro-ph].

[5] S. L. Adler, "Photon splitting and photon dispersion in a strong magnetic field," Annals Phys. 67, 599 (1971).

[6] T. Erber, "High-energy electromagnetic conversion processes in intense magnetic fields," Rev. Mod. Phys. 38, 626 (1966).

[7] E. Zavattini et al. [PVLAS Collaboration], "Experimental observation of optical rotation generated in vacuum by a magnetic field," Phys. Rev. Lett. 96, 110406 (2006) arXiv:hep-ex/0507107.

[8] E. Zavattini et al. [PVLAS Collaboration], "New PVLAS results and limits on magnetically induced optical rotation and ellipticity in vacuum," arXiv:0706.3419 [hep-ex].

[9] F. Aharonian et al., "Fast variability of tera-electron volt rays from the radio galaxy M87," Science 314, 1424 (2006).

[10] F. Krennrich et al., "Discovery of Spectral Variability of Markarian 421 at TeV Energies," Astrophys. J. 575, L9 (2002) arXiv:astro-ph/0207184.

[11] A. K. Konopelko, A. Mastichiadis, J. G. Kirk, O. C. de Jager and F. W. Stecker, "Modelling the TeV gamma-ray spectra of two low redshift AGNs: Mkn 501 and Mkn 421," Astrophys. J. 597, 851 (2003) arXiv:astro-ph/0302049.

[12] J. Kataoka et al., "High-Energy Emission from the TEV Blazar Markarian 501 during Multiwavelength Observations in 1996," Astrophys. J. 514, 138 (1999).

[13] H. E. S. S. collaboration, F. Aharonian, "Detection of VHE gamma-ray emission from the distant blazar 1ES 1101-232 with H.E.S.S. and broadband characterisation," arXiv:0705.2946 [astro-ph].

[14] C. C. Cheung, D. E. Harris and L. Stawarz, "Superluminal Radio Features in the M87 Jet and the Site of Flaring TeV Gamma-ray Emission," arXiv:0705.2448 [astro-ph].

[15] D. F. Torres, "Gamma-ray sources at high latitudes," arXiv:astro-ph/0308069

[16] M. Boettcher, "Modeling the emission processes in blazars," arXiv:astro-ph/0608713.

[17] D. Hooper and P. D. Serpico, "Detecting Axion-Like Particles With Gamma Ray Telescopes," arXiv:0706.3203 [hep-ph].

[18] F. Aharonian and A. Neronov, "TeV gamma rays from the galactic center," arXiv:astro-ph/0503354.

[19] F. Aharonian and A. Neronov, "High energy gamma rays from the massive black hole in the galactic center," Astrophys. J. 619, 306 (2005) arXiv:astro-ph/0408303.

[20] L. A. Anchordoqui, H. Goldberg, F. Halzen and T. J. Weiler, "Galactic point sources of $\mathrm{TeV}$ antineutrinos," Phys. Lett. B 593, 42 (2004) arXiv:astro-ph/0311002.

[21] A. Dupays and M. Roncadelli, "Discovering Light 
Pseudoscalar Bosons in Double-Pulsar Observations," arXiv:astro-ph/0612176.

[22] A. Dupays and M. Roncadelli, "Light pseudoscalar bosons, PVLAS and the double pulsar J0737-3039," arXiv:astro-ph/0612227.

[23] J. P. Vallée, "Observations of the Magnetic Fields Inside and Outside the Milky Way," Fundamentals of Cosmic Physics 19, 1 (1997).

[24] H. Ohno, S. Shibata, "The random magnetic field in the Galaxy," Mon. Not. R. Astron. Soc. 262, 953 (1993).

[25] J. L. Han, "Magnetic fields in our Galaxy: How much do we know? III. Progress in the last decade," Chin. J. Astron. Astrophys. 6, 211 (2006).

[26] E. Mortsell and A. Goobar, "Constraining photon axion oscillations using quasar spectra," JCAP 0304, 003 (2003) arXiv:astro-ph/0303081.

[27] A. De Angelis, O. Mansutti and M. Roncadelli, "AxionLike Particles, Cosmic Magnetic Fields and Gamma-Ray Astrophysics," arXiv:0707.2695 [astro-ph].

[28] M. Camenzind, "Relativistic Outflows form Active Galactic Nuclei," arXiv:astro-ph/0411573.

[29] G. B. Field and R. D. Rogers, "Radiation from Magnetized Accretion Disks in Active Galactic Nuclei", Astrophys. J. 403, 94 (1993).
[30] N. S. Kardashev, "Cosmic Supercollider", Mon. Not. R. Astron. Soc. 276, 515 (1995).

[31] A. Neronov and F. Aharonian, "Production of $\mathrm{TeV}$ gamma-radiation in the vicinity of the supermassive black hole in the giant radiogalaxy M87," arXiv:0704.3282 [astro-ph].

[32] M. Sikora, G. Madejski, R. Moderski, J. Poutanen, "Learning about Active Galactic Nucleus Jets from Spectral Properties of Blazars," Astrophys. J. 484, 108 (1997).

[33] P. Sreekumar, AIP Conference Proceedings, 510, 459 (2000).

[34] L. Stawarz, A. Siemiginowska, M. Ostrowski, M. Sikora, "On the Magnetic Field in the Kiloparsec-Scale Jet of Radio Galaxy M87," Astrophys. J. 626, 120 (2005).

[35] F. A. Aharonian, "Proton-synchrotron radiation of largescale jets in active galactic nuclei," Mon. Not. Roy. Astron. Soc. 332, 215 (2002).

[36] see, e.g., F. W. Stecker, "Exploring the Edge of the Stellar Universe with Gamma-Ray Observations," arXiv:astro-ph/0611455

[37] A. P. Lobanov, "Ultracompact jets in active galactic nuclei", Astron. Astrophys. 330, 79 (1998). 